\title{
Angiographic evaluation of the internal iliac artery branch in pelvic tumour patients: Diagnostic performance of multislice computed tomography angiography
}

\author{
LIN LI, KETONG WU, YANG LIU, HAIYANG LAI, ZHAOFEI ZENG and BO ZHANG
}

Department of Radiology, The Sixth Affiliated Hospital of Sun Yat-Sen University, Guangzhou, Guangdong 510655, P.R. China

Received April 17,2018; Accepted January 31, 2019

DOI: $10.3892 / \mathrm{ol} .2019 .10084$

\begin{abstract}
The aim of the present study was to explore the classification of the internal iliac artery (IIA) and the diagnostic value of the pelvic tumour-feeding artery by multislice computed tomography angiography (MSCTA) compared with digital subtraction angiography (DSA). A total of 43 patients with pelvic tumours were enrolled between January 2013 and August 2017. The classification of the IIA and the quality of the feeding artery of the pelvic tumours were analysed by Yamaki's classification (Groups A-D according to IIA branching) and the 5-point scoring system. The degree of feeding artery stenosis, caused by tumour compression or invasion, was analysed by a 4-point scoring system. The Wilcoxon signed-rank test was used to determine the vascular diagnostic quality identified by MSCTA and DSA. MSCTA of the pelvic arteries was successfully performed in all patients. The main classifications of the IIA were Group A, followed by Group C, then Group B and with no cases of Group D. There was no significant difference in the classification of the IIA between the left and right sides on MSCTA and DSA. The visualization quality of the IIA and its main branches showed excellent consistency, but the difference in the terminal branches of the feeding arteries in the pelvic tumours was statistically significant between MSCTA and DSA. MSCTA has great advantages in evaluating the classification of the IIA, the imaging quality evaluation of the IIA and its main branches, and in the evaluation of the pelvic tumour-feeding artery. However, in the display of the terminal arterial branches of the pelvic tumours, DSA remains irreplaceable, particularly in cases of interventional embolization.
\end{abstract}

Correspondence to: Professor Bo Zhang, Department of Radiology, The Sixth Affiliated Hospital of Sun Yat-Sen University, 26 Yuancun Erheng Road, Guangzhou, Guangdong 510655, P.R. China

E-mail: zhangbo19790616@163.com

Key words: multislice computed tomography angiography, digital subtraction angiography, internal iliac artery, feeding artery, pelvic tumour

\section{Introduction}

As one of the most important structures of the human body, the pelvic cavity has a complex anatomical structure, including multiple organs, fine vessels and nerves (1). These features suggest that a variety of diseases, including different types of tumours, may occur in the pelvic cavity. Pelvic tumours exhibit a wide range of origins and an abundant blood supply. The anatomical structure of the tumour-feeding arteries, as well as the collateral circulation and variation, may be more complex. In addition, a large tumour could compress the pelvic tissue and organs, leading to deformation and displacement of the anatomical structure of the pelvis (2). The location of a tumour and the feeding artery is therefore difficult to confirm $(1,3)$. Due to these features of the pelvic cavity, tracking the source of tumour-feeding arteries and determining a treatment programme, particularly an associated vascular interventional treatment, is essential.

As one of the main branches of the common iliac artery, the internal iliac artery (IIA) supplies the majority of the organs in the pelvic cavity, and it is believed that this artery is closely associated with the occurrence, development and treatment of pelvic diseases (3). With the widespread use of interventional embolization in the treatment of pelvic neoplasms, it is necessary for physicians to investigate the IIA and its pattern of division and branching to ensure any interventional procedure is secure and to avoid untargeted embolization. Nevertheless, due to numerous variations that exist in the anatomy of the IIA, for which there are also significant differences between sex and ethnic groups, there are no unified IIA classification criteria at present, although a number of studies have been performed on the classification of the IIA (4-7). The earliest systematic classification and description of the IIA was provided in 1928 by the Japanese scholar Adachi (8). The Adachi system classifies the distribution pattern of the IIA into 5 types with 8 groups based on a cadaver specimen study and remains in use today (8). Subsequently, Yamaki, a Japanese scholar, provided further research based on Adachi's classification and put forward Yamaki's classification (7), which is the most reproducible and simple classification for this complex vascular system, from clinical and imaging views (3). The studies by Adachi and Yamaki were performed on cadaver specimens, and the corresponding imaging evaluations, using different imaging modalities, are lacking. Although several 
previous studies have described the human pelvic vascular anatomy and its frequent variations from perspective imaging, interventional radiologists lack a simple model that can facilitate target branch identification (7,9-13).

At present, digital subtraction angiography (DSA) remains the gold standard for assessing blood vessels, particularly for smaller branches or vascular anastomosis. Additionally, DSA is also the primary route for determining the feeding artery of the tumour blood supply, which can be used to guide interventional therapy. However, DSA is an invasive procedure that is subject to inherent limitations, including vascular embolism, vascular injury and high cost. With the development of radiology technology, multislice computed tomography angiography (MSCTA) is an important technique that is increasingly used in vascular imaging studies for its characteristic merits, including observing vascular lesions in various directions and investigating the variation of blood flow at different stages of vascular lesions through CT post-processing technology $(6,14-16)$.

Although the diagnostic performance of MSCTA has been proven to be superior to that of DSA in certain fields $(17,18)$, there are few reports detailing the anatomy of the IIA and its branches by MSCTA, as well as assessments of the diagnostic quality of the feeding arteries in pelvic tumours. Therefore, the aim of the present study was to explore the anatomical structure and classification characteristics of the IIA, and to estimate the diagnostic value for preoperative assessment of patients with pelvic tumours by MSCTA compared with DSA.

\section{Materials and methods}

Study population. Between January 2013 and August 2017, a total of 43 patients ( 27 male and 16 female) with pelvic tumours who has been assessed in The Sixth Affiliated Hospital of Sun Yat-sen University (Guangzhou, Guangdong, China) were enrolled in the present study. MSCTA and DSA examinations and intervention therapy were performed for all patients. The mean age of the patients was 52.7 years (range, 24-91 years). Radiological imaging and medical records were reviewed to obtain valuable information, including clinical characteristics, visualization quality, classification of the IIA and terminal branches of the pelvic tumour-feeding artery. The diagnosis of these patients was routinely confirmed by two senior pathologists at The Sixth Affiliated Hospital of Sun Yat-sen University by histopathology and immunohistochemistry (IHC) of biopsy or surgical samples. There were 9 cases of refractory bladder bleeding and 4 cases of rectal bleeding due to tumour invasion. The main manifestations of the remaining patients included abdominal pain and distension, an abdominal mass and complicated intestinal obstruction.

Patients with the following criteria were excluded: Iodine allergy, severe coagulation disorders with an evident bleeding tendency, severe arteriosclerosis, vital organ failure, tumour cachexia, high fever, infection, female menstrual period and pregnancy. The study protocol was approved by the Institutional Ethics Review Board of The Sixth Affiliated Hospital of Sun Yat-Sen University and complies with the ethical principles of the Declaration of Helsinki. Informed consent was obtained from all individual participants included in the study.
IHC staining procedure. Tissue blocks were fixed in $10 \%$ formalin and embedded in paraffin, and were then sliced into several sections at a thickness of $4 \mu \mathrm{m}$ prior to IHC staining. Briefly, the sections were dewaxed in xylene and hydrated with a graded alcohol series, and were then washed with PBS (pH 7.4) three times, each time for $3 \mathrm{~min}$. The sections were placed in freshly-prepared boiled citrate buffer $(\mathrm{pH}$ 6.0) for antigen retrieval at $95^{\circ} \mathrm{C}$ from $5 \mathrm{~min}$. The sections were treated with $3 \% \mathrm{H}_{2} \mathrm{O}_{2}$ in $0.1 \mathrm{M}$ PBS to block endogenous peroxidase activity at room temperature for $30 \mathrm{~min}$. Following washing with PBS, each slide was incubated with the appropriate primary antibody overnight at $4^{\circ} \mathrm{C}$. PBS alone was used as the blank control. Slides were washed three times following incubation with the appropriate secondary antibody in $0.1 \mathrm{M}$ PBS ( $\mathrm{pH}$ 7.4) for $30 \mathrm{~min}$ at room temperature. The IHC reaction was visualized with a light microscope (magnification, x200 or x400) following 3,3'-diaminobenzidine chromogen staining for 5-10 $\mathrm{min}$ at room temperature and hematoxylin counterstaining for $5 \mathrm{~min}$ at room temperature.

MSCTA imaging and image reconstruction protocol. Patients fasted for 4-6 h prior to MSCTA examination. A 128- or 640-row multidetector spiral CT scanner (Toshiba Aquilion ONE; Canon, Inc., Tokyo, Japan) was used. The patient was placed in a supine position on the scanner table. The range of the scan was from the lower edge of the fourth lumbar spine to the upper femur. The scan parameters were $120 \mathrm{kV}$ and $450 \mathrm{mAs}$. Each patient received a single CT scan. Power injection settings were as follows: Iodine contrast agent volume, $100 \mathrm{ml}$ (iopromide injection, $370 \mathrm{mg} / \mathrm{ml}$ ); and injection rate, $4 \mathrm{ml} / \mathrm{sec}$, injected by the elbow vein, followed by an injection of $20 \mathrm{ml}$ physiological saline by the same method. The bolus injection was used to measure the CT value in the abdominal aorta (above the renal arteries), which acted as the region of interest (ROI). The MSCTA scan (arterial phase, venous phase and delay period) automatically started when the CT value in the ROI reached 100 Houndsfield units and was then delayed $6 \mathrm{sec}$. The acquired data was interpreted by reading the axial reformats at $\leq 1.0 \mathrm{~mm}$ in thickness, which allowed study of the vascular anatomy. Post-processing was performed using StartVitrea 6.6 post-processing software (Canon, Inc.). Multi-planner reformation, maximum intensity projections (MIPs) and volume rendering were used with three-dimensional (3D) reconstructions. Satisfactory MSCTA images were reconstructed according to these 3 reconstruction techniques by adjusting the different thresholds and patterns.

DSA protocol. A DSA instrument (INNOVA 3100; GE Healthcare, Chicago, IL, USA) was used. A right transfemoral approach was used for artery access. Initially, the 2 pelvic common iliac arteries were visualised by performing digital angiography in the abdominal aorta (injection volume, $20 \mathrm{ml}$; injection rate, $15 \mathrm{ml} / \mathrm{sec}$ ). Subsequently, the two IIAs were catheterised for imaging. The contralateral (usually the left) IIA was initially catheterised and visualised by performing digital angiography in the artery origin in the neutral position and repeating in the contralateral oblique and ipsilateral oblique positions (injection volume, $10 \mathrm{ml}$; injection rate, $5 \mathrm{ml} / \mathrm{sec}$ ). The right IIA was visualised by the same method following catheterisation. 
Image evaluation. All MSCTA and DSA images were assessed by 2 senior radiologists with 5 and 3 years of experience in the field of CT vascular imaging and angiography, respectively. The 2 readers were blinded to the diagnostic patient information and to the results of each other's analysis. The cases were randomly reviewed and the reading interval between MSCTA and DSA images was 7-10 days. The pelvic arterial 3D vascular network was constructed, and the anatomical structures of the IIA were contained in the evaluation and assessed by the classification criteria of Yamaki et al (7). The mode of branching of the IIA was classified into 4 groups. Group A: The IIA divided into 2 branches, the superior gluteal artery, (posterior division) and the common trunk of the inferior gluteal and internal pudendal arteries (anterior division). Group B: The IIA divided into 2 branches, the common gluteal trunk (posterior division) of the superior gluteal and inferior gluteal arteries, and the internal pudendal artery (anterior division). Group C: The IIA simultaneously divided into 3 major branches, the internal pudendal, inferior gluteal and superior gluteal arteries. Group D: The IIA divided into the inferior gluteal artery (posterior division), the common trunk of the superior gluteal and internal pudendal arteries (anterior division).

Regarding the quality evaluation of IIA and its branches, 4 main branches were counted as 4 anatomical segments, according to Yamaki's classification, and they were separately evaluated on MSCTA and DSA images.

The readers evaluated the images according to a 5-point scoring system, which was modified from Danias et al (19) and Pfeil et al (20) as follows: 1 point, non-diagnostic; 2 points, poor quality, vessel border was suspected, but not clearly visible, and vessel segments definable, but with significant blurring or artefacts; 3 points, moderate quality, sharpness of the vessel border was insufficient, but vessel segments clearly definable with moderate blurring or artefacts; 4 points, good quality, good sharpness of the vessel border and diagnostic information available with minimal blurring or artefacts; and 5 points, excellent diagnostic quality without blurring or artefacts, and with sharply defined vessel borders.

In addition, the 2 readers tracked the feeding arteries of the pelvic tumours and assessed the imaging quality of the trunk and terminal branches of the tumour-feeding artery according to the 5-point scoring system. Due to the complexity of the type of tumour and the branches of the blood supply artery, it was difficult to define the terminal branch of the feeding artery. To accurately evaluate the imaging quality between the two examinations, the 2 readers defined the terminal branch of the feeding artery as the vascular segment that contacted or entered the tumour subsequent to the feeding artery.

The existence of pelvic tumours may cause compression or stenosis of the feeding arteries. Changes in the arterial diameter caused by the tumours were evaluated with source images and reconstructed images (mainly MIP images) of each tumour in the imaging modes by the 2 readers. The stenosis was recorded as follows: 0, Artery free from tumour; 1, artery displaced, but not narrowed by tumour; 2, tumour narrowing artery $<50 \%$; and 3 , tumour narrowing artery $\geq 50 \%$.

Statistical analysis. Data were processed using SPSS 20.0 software (IBM Corp., Armonk, NY, USA). Measurement data are expressed as the mean \pm standard deviation and count data are expressed as a percentage. The 2 sample rates were compared using the $\chi^{2}$ test for a $2 \times 4$ data table. The differences in the vascular image quality of the IIA and the visualization quality of the tumour-feeding arteries between the two imaging modes were determined by the Wilcoxon signed-rank test. A $\kappa$ test was used to analyse the conformity between the two imaging modes. $\kappa$ coefficient values were interpreted as excellent consistency $\geq 0.80$, good consistency $0.61-0.80$, medium $0.41-0.60$ and poor $\leq 0.40$ (21). $\mathrm{P}<0.05$ was considered to indicate that a difference was statistically significant.

\section{Results}

The 3D digital models of pelvic arteries in all 43 pelvic tumour patients were successfully constructed. These tumours included rectal cancer $(n=15)$, cervical cancer $(n=6)$, prostate cancer $(n=4)$, gastrointestinal stromal tumours in the rectum $(n=3)$, liposarcoma $(n=3)$, malignant solitary fibrous tumours $(n=2)$, Ewing sarcoma $(n=2)$, neurofibrosarcoma $(n=1)$, sigmoid colon cancer $(n=1)$, anal canal carcinoma $(n=1)$, uterine leiomyosarcoma $(n=1)$, ovarian mucinous cystadenocarcinoma $(n=1)$, bladder cancer $(n=1)$, a sacrococcygeal neurogenic spindle cell tumour $(n=1)$ and a gastric cancer pelvic metastatic tumour $(n=1)$. The branching patterns of the IIA of 86 pelvic sides were reviewed with MSCTA and DSA, based on the 4 groups of Yamaki's classification. No other special types were identified in the present study. Based on images acquired by MSCTA, 64 pelvic sides were classified as Group A (74.4\%) (Fig. 1), 8 as Group B (9.3\%) (Fig. 2) and 14 as Group C (16.3\%) (Fig. 3), with no cases in Group D. Of the 43 patients, 26 had the same type of bilateral IIA. Additionally, no significant difference was observed between the right and left sides in the classification of the IIA ( $\mathrm{P}=0.73$; Table I). The classifications of 86 pelvic sides of the IIA were also assessed in images acquired by DSA. A total of 63 pelvic sides were classified as Group A (73.3\%), 7 as Group B (8.1\%) and 16 as Group C (18.6\%), with no cases of Group D. Of the 43 patients, 23 had the same type of bilateral IIA, and no significant difference was observed between the right and left sides in the classification of the IIA ( $\mathrm{P}=0.95$; Table II). These results demonstrated an excellent consistency between MSCTA and DSA with regard to the classification of the IIA $(\kappa=0.81)$ (Table III).

Regarding the evaluation of the quality of images of the IIA and its branches, a total of 430 vascular anatomical segments of the IIAs and their branches were separately evaluated on MSCTA and DSA images. The 2 readers carefully analysed each arterial segment and scored and summarized the imaging quality (Table IV). The results indicated that the overall imaging quality of DSA was slightly higher compared with that of MSCTA, but the difference between the means was not statistically significant (DSA, 4.14 \pm 0.94 ; MSCTA, $4.13 \pm 0.95 ; \mathrm{P}=0.09)$. In addition, an excellent image quality was shown between the two imaging modes $(\kappa=0.94 \pm 0.06)$ and the 2 readers achieved excellent consistency in the assessment of the vascular imaging quality in the MSCTA and DSA images $\left(\kappa_{\mathrm{MSCTA}}=0.87 \pm 0.10, \kappa_{\mathrm{DSA}}=0.88 \pm 0.09\right)$.

As several different types of pelvic neoplasms were included in the present study, the arteries supplying blood to the tumours were tracked and identified on DSA and MSCTA. 


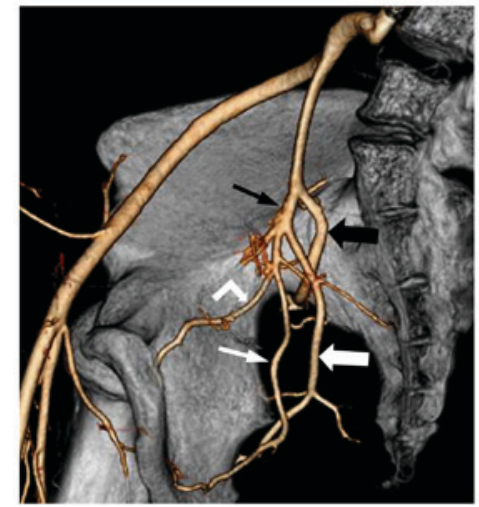

Figure 1. Pelvic liposarcoma and a Group A bifurcation of the right IIA in a 31-year-old male patient. The three-dimensional volume-rendered, reformatted computed tomography angiogram shows the right IIA dividing into 2 branches: Superior gluteal artery (thick black arrow) and common trunk (thin black arrow), which bifurcates into the inferior gluteal artery (thick white arrow) and the internal pudendal artery (thin white arrow). The angled arrow indicates the obturator artery arising from the internal pudendal artery (angled thin white arrow). IIA, internal iliac artery.

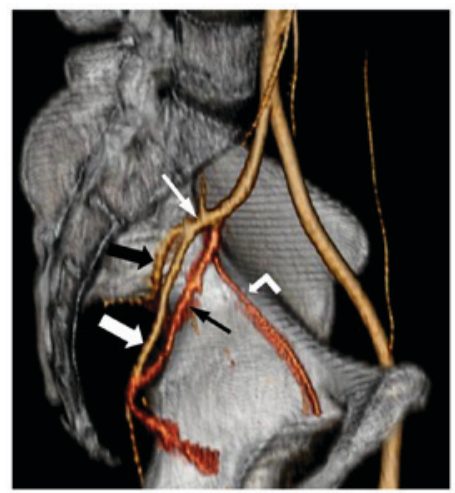

Figure 2. Rectal carcinoma and a Group B bifurcation of the left IIA in a 24-year-old male patient. The three-dimensional volume-rendered, reformatted computed tomography angiogram shows the left IIA dividing into 2 branches: The internal pudendal artery (thin black arrow) and the common gluteal trunk (thin white arrow) of the superior gluteal artery (thick black arrow), and the inferior gluteal artery (thick white arrow). The angled arrow indicates the obturator artery arising from the internal pudendal artery (angled thin white arrow). IIA, internal iliac artery.

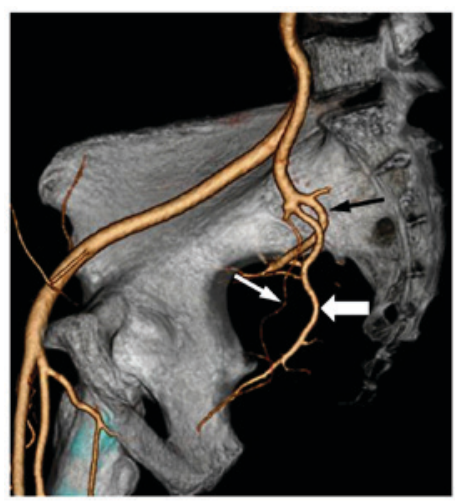

Figure 3. Uterine leiomyosarcoma and a Group $\mathrm{C}$ bifurcation of the right IIA in a 49-year-old female patient. The three-dimensional volume-rendered, reformatted computed tomography angiogram shows the IIA dividing simultaneously into the superior gluteal (thin black arrow), inferior gluteal (thick white arrow) and internal pudendal arteries (thin white arrow). There is no apparent obturator artery arising from the branches of the IIA. IIA, internal iliac artery.
There was no difference in the types of feeding arteries between the two examinations, and the accuracy of MSCTA in diagnosing the type and quantity of feeding arteries was $100 \%$ (Fig. 4), which is consistent with the results using DSA.

To evaluate the ability of MSCTA and DSA to display the terminal branches of the feeding artery, the 2 readers tracked the feeding arteries of the pelvic tumours and assessed the imaging quality of the trunk and terminal branches of the tumour-feeding artery according to the 5-point scoring system. The results demonstrated that the two imaging modes had good consistency in displaying the main trunk of the feeding artery $(\kappa=0.67)$. However, the consistency of MSCTA and DSA was poor in displaying the terminal small branches of the feeding arteries $\left(\kappa=0.14 ; \chi^{2}=14.29 ; \mathrm{P}=0.027\right)$. Therefore, the difference was statistically significant between the MSCTA and DSA images for showing the terminal branches of feeding arteries in pelvic tumours (Fig. 5).

Finally, the degree of arterial stenosis caused by pelvic tumours on the imaging modes was summarized by the 2 readers. The internal consistency of MSCTA and DSA was excellent for each of the 2 readers (reader $1, \kappa=0.87$; reader 2 , $\kappa=0.81$ ). Excellent consistency between the 2 readers was also obtained on MSCTA and DSA (MSCTA, $\kappa=0.81$; DSA, $\kappa=0.97)$.

\section{Discussion}

As a non-invasive and comprehensive method for the imaging of the majority of the major vessels in the body, MSCTA may provide visualiszation of the $3 \mathrm{D}$ spatial association between the tumour, tumour-feeding arteries, and adjacent organs and large blood vessels by using post-processing reconstruction technology $(14,22)$. As the gold standard for vascular display, DSA can visually display and observe the shape of blood vessels and the location of lesions. The technique has a large advantage in tracking the vascular path and showing the image in real time, and associated treatments can be performed while conducting a diagnosis. In addition, the radiation dose of the minimally invasive examination of DSA may be slightly higher compared with that of MSCTA, but remains in the safe range. Several studies reported that the sensitivity and specificity of MSCTA in the diagnosis of certain vascular diseases was approximately the same as DSA $(23,24)$. However, the absence of data on the non-invasive examination of vascular anatomy of complex pelvic tumours, particularly data on the preoperative assessment of the vascular and haemodynamic parameters, may complicate the treatment and prognosis of tumours.

In the present study, MSCTA and DSA were used to investigate the classification of the IIA, which is the main feeding artery of the pelvic cavity with dense branches. Although there is no uniform classification of the IIA at present (4), Yamaki's classification is the most commonly used classification criteria. In the study by Yamaki et al (7), 645 pelvic sides of the IIA were typed by autopsy, and Group A was considered the basic branching pattern of the IIA, as it was the most frequent (79.5\%), followed by Group B (15.0\%), while Group C and Group D occurred at rates of 5.3 and $0.2 \%$, respectively. In the present study, the main classification of the IIA was also Group A, but Group B was less frequent compared with Group C, and no patients were classified as Group D. The 
Table I. Classification of the internal iliac arteries in the left and right sides by multislice computed tomography angiography.

\begin{tabular}{|c|c|c|c|c|c|c|c|}
\hline Side & Total, $\mathrm{n}$ & Group A, n (\%) & Group B, n (\%) & Group C, n (\%) & Group D, n (\%) & $\chi^{2}$ & P-value \\
\hline Right & 43 & $33(76.8)$ & $5(11.6)$ & $5(11.6)$ & $0(0.0)$ & & \\
\hline Left & 43 & $31(72.1)$ & $3(7.0)$ & $9(20.9)$ & $0(0.0)$ & & \\
\hline Total & 86 & $64(74.4)$ & $8(9.3)$ & $14(16.3)$ & $0(0.0)$ & 2.0 & 0.73 \\
\hline
\end{tabular}

Table II. Classification of the internal iliac arteries in the left and right sides by digital subtraction angiography.

\begin{tabular}{|c|c|c|c|c|c|c|c|}
\hline Side & Total, $\mathrm{n}$ & Group A, n (\%) & Group B, n (\%) & Group C, n (\%) & Group D, n (\%) & $\chi^{2}$ & $\mathrm{P}$-value \\
\hline Right & 43 & $33(76.8)$ & $5(11.6)$ & $5(11.6)$ & $0(0.0)$ & & \\
\hline Left & 43 & $30(72.1)$ & $2(4.7)$ & $11(23.2)$ & $0(0.0)$ & & \\
\hline Total & 86 & $63(73.3)$ & $7(8.1)$ & $16(18.6)$ & $0(0.0)$ & 0.71 & 0.95 \\
\hline
\end{tabular}

Table III. Consistency analysis between classification of the internal iliac arteries by MSCTA and DSA.

\begin{tabular}{lcccc}
\hline Technique & Group A, $\mathrm{n}$ & Group B, $\mathrm{n}$ & Group C, $\mathrm{n}$ & Group D, $\mathrm{n}$ \\
\hline MSCTA & 64 & 8 & 14 & 0 \\
DSA & 63 & 7 & 16 & 0 \\
\end{tabular}

An excellent consistency was identified $(\kappa=0.81)$. MSCTA, multislice computed tomography angiography; DSA, digital subtraction angiography

Table IV. Imaging quality of 430 vascular anatomical segments of the IIAs evaluated on CTA and DSA images.

\begin{tabular}{|c|c|c|c|c|c|c|}
\hline \multirow[b]{2}{*}{ Vessel segment } & \multicolumn{3}{|c|}{ CTA } & \multicolumn{3}{|c|}{ DSA } \\
\hline & Reader 1 & Reader 2 & $\kappa$ & Reader 1 & Reader 2 & $\kappa$ \\
\hline IIA & $5.00 \pm 0.00$ & $5.00 \pm 0.00$ & 1.00 & $5.00 \pm 0.00$ & $5.00 \pm 0.00$ & 1.00 \\
\hline Superior gluteal artery & $4.53 \pm 0.57$ & $4.52 \pm 0.63$ & 0.80 & $4.57 \pm 0.56$ & $4.57 \pm 0.61$ & 0.84 \\
\hline Inferior gluteal artery & $4.27 \pm 0.71$ & $4.27 \pm 0.71$ & 0.84 & $4.26 \pm 0.69$ & $4.28 \pm 0.70$ & 0.79 \\
\hline Obturator artery & $3.10 \pm 1.01$ & $3.06 \pm 1.01$ & 0.79 & $3.09 \pm 0.98$ & $3.09 \pm 0.98$ & 0.82 \\
\hline Internal pudendal artery & $3.76 \pm 0.67$ & $3.78 \pm 0.68$ & 0.80 & $3.80 \pm 0.67$ & $3.78 \pm 0.66$ & 0.80 \\
\hline Mean \pm SD & $4.13 \pm 0.94$ & $4.13 \pm 0.96$ & $0.87 \pm 0.10$ & $4.14 \pm 0.93$ & $4.14 \pm 0.94$ & $0.88 \pm 0.09$ \\
\hline
\end{tabular}

IIA, internal iliac artery; CTA, computed tomography angiography; DSA, digital subtraction angiography; SD, standard deviation.

relatively small number of samples in the present study may be the cause of these unexpected results. In general, the dominant classification was Group A, and there was no significant difference in the classification of the IIA between the left and right sides, in MSCTA and DSA images $(\mathrm{P}=0.95)$, which was consistent with the study by Yamaki et al (7).

In addition, the two imaging modes had excellent consistency in the IIA classification $(\kappa=0.81)$. In the present study, the total imaging quality of the IIA and its branches on MSCTA appeared slightly lower compared with that on DSA, but the difference between them was not statistically significant $(\mathrm{P}=0.09)$.
Regarding the assessment of the supply artery and small terminal branches in the pelvic tumour, images acquired by MSCTA clearly revealed the type and number of feeding arteries in the pelvic tumours, with no difference between the two examinations. Additionally, the accuracy of MSCTA in diagnosing the type and imaging quantity of the feeding arteries was $100 \%$, which was consistent with DSA. MSCTA and DSA demonstrated good consistency in visualising the main trunk of the feeding artery $(\kappa=0.67)$. However, the two imaging modes exhibited poor consistency and a significant difference in the assessment of the small terminal branches of the feeding arteries $(\kappa=0.14 ; \mathrm{P}=0.027)$. These results may 

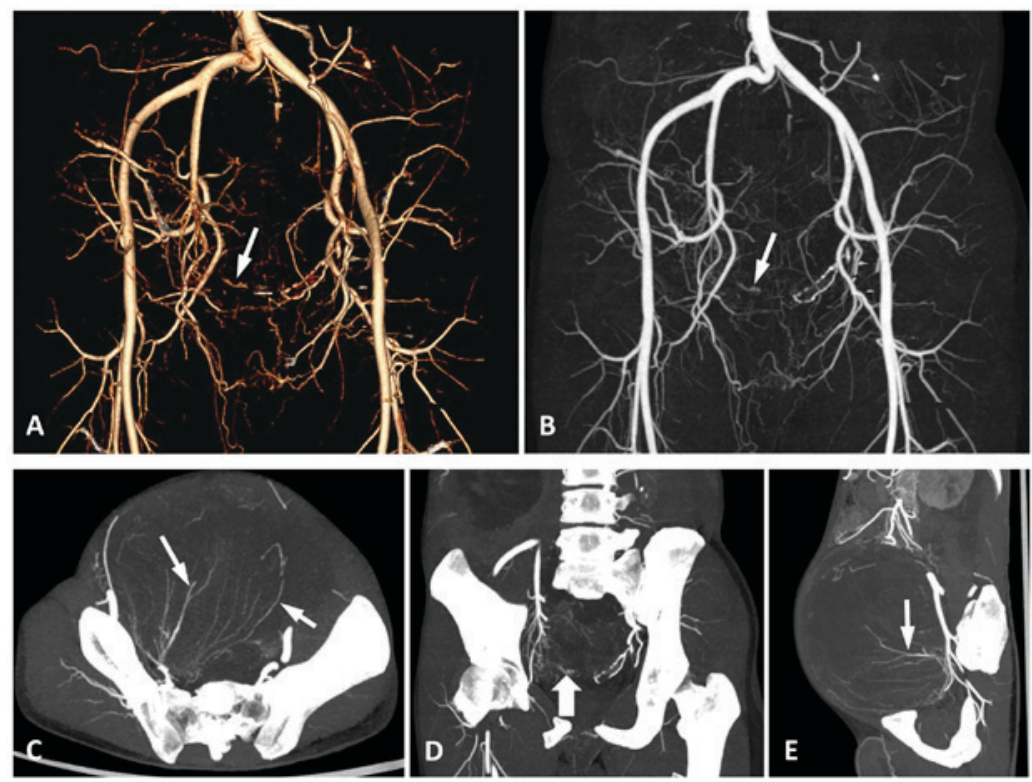

Figure 4. Pelvic liposarcoma in a 31-year-old male patient. (A) The volume reconstruction and (B) thick layer MIP images show the three-dimensional structure of the vascular anatomy in the pelvis and the tumour-feeding arteries (white arrows). (C) Axial, (D) coronal and (E) sagittal thin-layer MIP images show the tortuous and fine feeding artery network (thin white arrows) displayed with interruption of the supply artery (thick white arrow). MIP, maximum intensity projection.
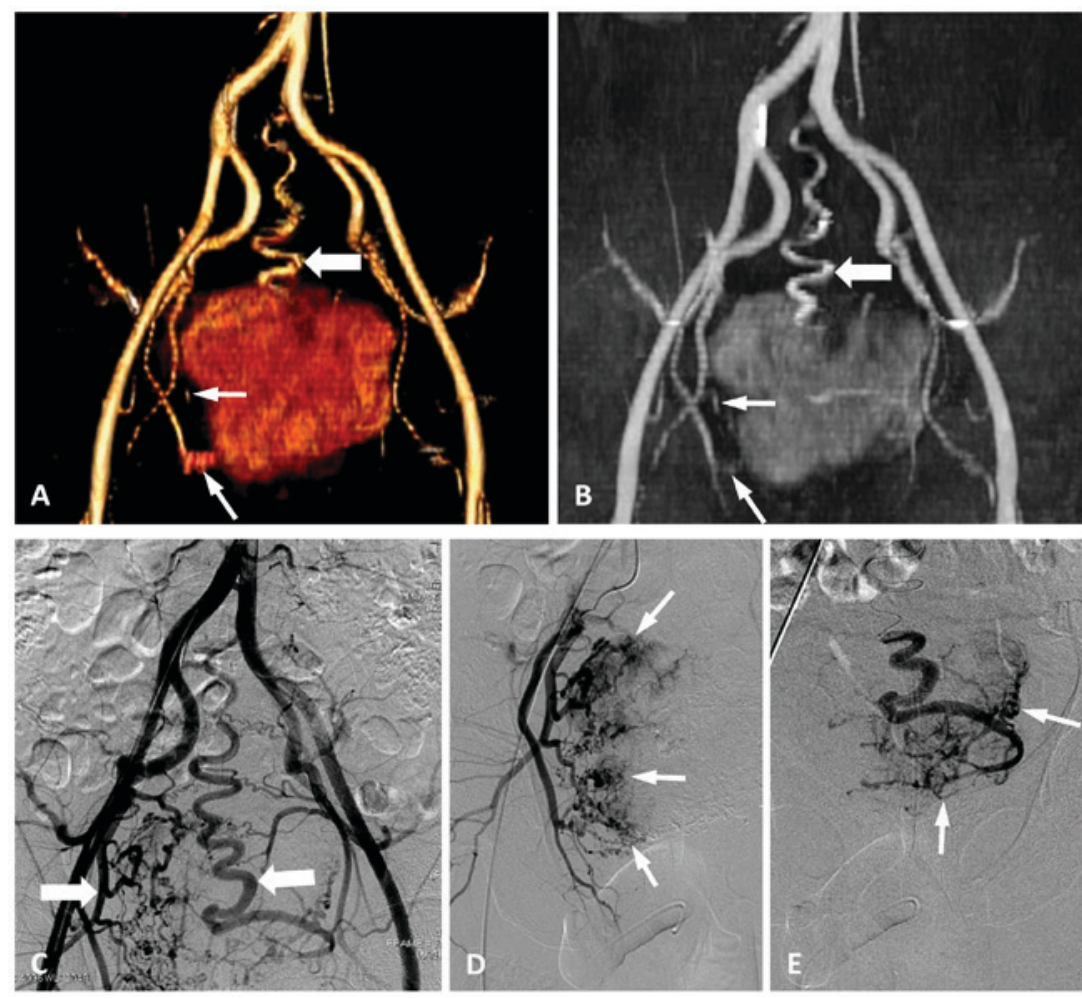

Figure 5. Malignant solitary fibrous tumour in a 44-year-old male patient. (A) The multislice computed tomography angiography volume reconstruction image and (B) maximum intensity projection image show the tortuous and irregular tumour-feeding arteries (thin white arrows), and the median sacral artery (thick white arrow), which is particularly apparent. In addition to the main branches of tumour-feeding arteries, (C, thick white arrow) the tortuous terminal arterial branches, (D, thin white arrow) right internal iliac artery and (E, thin white arrow) middle sacral artery are also clearly displayed in the superselective digital subtraction angiography images.

indicate that DSA is more useful compared with MSCTA in the evaluation of the small branches, a finding that has been reflected in a previous study (3). In addition, the extent of arterial invasion by pelvic tumours is of interest to clinicians, particularly oncologists. The internal consistency was excellent between the two imaging modes and the 2 readers. MSCTA appears to be an ideal alternative to DSA in assessing arterial invasion caused by pelvic tumours. 
The rapid development of pelvic vascular interventional therapy based on the arterial system has led to its widespread use in women with hysteromyoma and postpartum haemorrhage associated with pelvic vascular injury, in men with benign prostatic hyperplasia and in patients with advanced pelvic tumours that were not suitable for surgery, as well as in other candidates (25-27). Selective arterial cannulation is necessary for vascular interventional therapy. In addition, the IIA and its branches are the main blood supply for pelvic tumours, so it is important to understand the classification and systematic anatomical structure of the IIA, and to confirm the arterial source of the blood supply prior to treatment $(28,29)$.

Imaging of the IIA in 3D using MSCTA and post-processing software demonstrated that MSCTA may replace DSA in the evaluation of the iliac vascular classification to a certain extent (3). For pelvic intervention, the introduction of high-quality non-invasive imaging techniques, including MSCTA, could facilitate the study of the classification and branches of the IIA by interventional radiologists in more detail, with the aim of positioning the target vessel correctly, improving the accuracy of selective cannulation and greatly reducing the procedure time. In addition, for surgery and minimally invasive surgery, the construction of the 3D anatomy of the pelvic vessels prior to surgery may improve the accuracy of the surgery and reduce intraoperative and postoperative bleeding, and other serious complications.

Additionally, MSCTA can successfully evaluate the types of arteries feeding the tumours. The present study revealed that there were no significant differences between MSCTA and DSA in visualising the main trunk of the feeding arteries in pelvic tumours. However, MSCTA was less sensitive compared with DSA in displaying the terminal small branches of tumour-feeding arteries. DSA is an invasive procedure, which is usually used prior to pelvic selective arterial embolization; the bilateral iliac vessels cannot be clearly displayed simultaneously on it, which means that DSA cannot be used as a routine assessment for identifying the feeding arteries of pelvic tumours. Preoperative MSCTA enables interventional radiologists to achieve more information about the feeding arteries in pelvic tumours, including origin, form and branches, particularly in older patients where iliac atherosclerotic lesions may prohibit vascular access.

The present study had several limitations. Firstly, the data was analysed retrospectively and, as such, the study was subject to the inherent limitations of retrospective studies. Secondly, the classification of the IIA was divided based on the main branch of the IIA, ignoring the influence of its small branches for classification. Finally, the present single-centre preliminary study had a small sample size, and the imaging quality of the IIA and the feeding arteries was assessed by 2 radiologists, which may cause bias to a certain degree. Therefore, a multicentre study or a systematic meta-analysis may be required to conquer these limitations.

In conclusion, MSCTA demonstrated excellent consistency with DSA in the classification of the IIA, the imaging quality evaluation of the IIA and its branches, and in the evaluation of the pelvic tumour-feeding artery, which is expected to provide an anatomical basis for pelvic interventional therapy and surgical treatment. However, in the visualisation of the terminal arterial branches of the pelvic tumour, DSA remains irreplaceable, particularly in cases of interventional embolization.

\section{Acknowledgements}

Not applicable.

\section{Funding}

This study was supported by grants from the National Natural Science Foundation of China (no. 81301978), the Ph.D. Programs Foundation of Ministry of Education of China (no. 20130171120105) and the Young Teacher Cultivation Project of Sun Yat-Sen University (no. 13YKPY39).

\section{Availability of data and materials}

The datasets used and/or analyzed during the present study are available from the corresponding author on reasonable request.

\section{Authors' contributions}

LL and BZ drafted this manuscript. LL, KW and BZ analyzed the imaging data and collected the data. YL, HL and ZZ assisted with statistical analysis. All authors read and approved the final manuscript.

\section{Ethics approval and consent to participate}

The study protocol was approved by the Institutional Ethics Review Board of The Sixth Affiliated Hospital of Sun Yat-Sen University (Guangzhou, China). Written informed consent for this study was obtained from each patient.

\section{Patient consent for publication}

Not applicable.

\section{Competing interests}

The authors declare that they have no competing interests.

\section{References}

1. Ding HM, Yin ZX, Zhou XB, Li YB, Tang ML, Chen SH, Xu DC and Zhong SZ: Three-dimensional visualization of pelvic vascularity. Surg Radiol Anat 30: 437-442, 2008.

2. Levy AD, Manning MA, Al-Refaie WB and Miettinen MM: Soft-tissue sarcomas of the abdomen and pelvis: Radiologic-pathologic features, part 1-common sarcomas: From the radiologic pathology archives. Radiographics 37: 462-483, 2017.

3. Bilhim T, Casal D, Furtado A, Pais D, O'Neill JE and Pisco JM: Branching patterns of the male internal iliac artery: Imaging findings. Surg Radiol Anat 33: 151-159, 2011.

4. Chantalat E, Merigot O, Chaynes P, Lauwers F, Delchier MC and Rimailho J: Radiological anatomical study of the origin of the uterine artery. Surg Radiol Anat 36: 1093-1099, 2014.

5. Naguib NN, Nour-Eldin NE, Hammerstingl RM, Lehnert T, Floeter J, Zangos S and Vogl TJ: Three-dimensional reconstructed contrast-enhanced MR angiography for internal iliac artery branch visualization before uterine artery embolization. J Vasc Interv Radiol 19: 1569-1575, 2008.

6. Selvaraj L and Sundaramurthi I: Study of normal branching pattern of the coeliac trunk and its variations using CT angiography. J Clin Diagn Res 9: AC01-AC04, 2015.

7. Yamaki K, Saga T, Doi Y, Aida K and Yoshizuka M: A statistical study of the branching of the human internal iliac artery. Kurume Med J 45: 333-340, 1998. 
8. Sakthivelavan S, Aristotle S, Sivanandan A, Sendiladibban S and Felicia Jebakani C: Variability in the branching pattern of the internal iliac artery in Indian population and its clinical importance. Anat Res Int 2014: 597103, 2014.

9. Bilhim T, Pereira JA, Fernandes L, Rio Tinto H and Pisco JM: Angiographic anatomy of the male pelvic arteries. AJR Am J Roentgenol 203: W373-W382, 2014.

10. Mori K, Saida T, Shibuya Y, Takahashi N, Shiigai M, Osada K, Tanaka N and Minami M: Assessment of uterine and ovarian arteries before uterine artery embolization: Advantages conferred by unenhanced MR angiography. Radiology 255: 467-475, 2010.

11. Rott $G$ and Boecker F: The extremely rare vascular variant of a segmental duplicated uterine artery and its relevance for the interventionist and gynecologist: A case report. J Med Case Rep 10: 162,2016

12. de Assis AM, Moreira AM, de Paula Rodrigues VC, Harward SH, Antunes AA, Srougi M and Carnevale FC: Pelvic arterial anatomy relevant to prostatic artery embolisation and proposal for angiographic classification. Cardiovasc Intervent Radiol 38: $855-861,2015$.

13. Bilhim T, Pisco JM, Rio Tinto H, Fernandes L, Pinheiro LC Furtado A, Casal D, Duarte M, Pereira J, Oliveira AG and O'Neill JE: Prostatic arterial supply: Anatomic and imaging findings relevant for selective arterial embolization. J Vasc Interv Radiol 23: 1403-1415, 2012.

14. Hu HJ, Huang YW and Zhu YC: Tumor feeding artery reconstruction with multislice spiral CT in the diagnosis of pelvic tumors of unknown origin. Diagn Interv Radiol 20: 9-16, 2014.

15. Wang MQ, Duan F, Yuan K, Zhang GD, Yan J and Wang Y: Benign prostatic hyperplasia: Cone-beam CT in conjunction with DSA for identifying prostatic arterial anatomy. Radiology 282: 271-280, 2017.

16. Lee JH, Jeong YK, Park JK and Hwang JC: 'Ovarian vascular pedicle' sign revealing organ of origin of a pelvic mass lesion on helical CT. AJR Am J Roentgenol 181: 131-137, 2003.

17. Ota H, Takase K, Igarashi K, Chiba Y, Haga K, Saito H and Takahashi S: MDCT compared with digital subtraction angiography for assessment of lower extremity arterial occlusive disease: Importance of reviewing cross-sectional images. AJR Am J Roentgenol 182: 201-209, 2004

18. Duffis EJ, Jethwa P, Gupta G, Bonello K, Gandhi CD and Prestigiacomo CJ: Accuracy of computed tomographic angiography compared to digital subtraction angiography in the diagnosis of intracranial stenosis and its impact on clinical decision-making. J Stroke Cerebrovasc Dis 22: 1013-1017, 2013.

19. Danias PG, McConnell MV, Khasgiwala VC, Chuang ML, Edelman RR and Manning WJ: Prospective navigator correction of image position for coronary MR angiography. Radiology 203: 733-736, 1997.
20. Pfeil A, Betge S, Poehlmann G, Boettcher J, Drescher R, Malich A, Wolf G, Mentzel HJ and Hansch A: Magnetic resonance VIBE venography using the blood pool contrast agent gadofosveset trisodium-An interrater reliability study. Eur J Radiol 81: 547-552, 2012.

21. Landis JR and Koch GG: The measurement of observer agreement for categorical data. Biometrics 33: 159-174, 1977.

22. Farghadani M, Momeni M, Hekmatnia A, Momeni F and Baradaran Mahdavi MM: Anatomical variation of celiac axis, superior mesenteric artery, and hepatic artery: Evaluation with multidetector computed tomography angiography. J Res Med Sci 21: 129, 2016.

23. Schernthaner R, Stadler A, Lomoschitz F, Weber M, Fleischmann D, Lammer J and Loewe Ch: Multidetector CT angiography in the assessment of peripheral arterial occlusive disease: Accuracy in detecting the severity, number, and length of stenoses. Eur Radiol 18: 665-671, 2008.

24. Delgado Almandoz JE, Romero JM, Pomerantz SR and Lev MH: Computed tomography angiography of the carotid and cerebral circulation. Radiol Clin North Am 48: 265-281, 2010.

25. Fang JF, Shih LY, Wong YC, Lin BC and Hsu YP: Repeat transcatheter arterial embolization for the management of pelvic arterial hemorrhage. J Trauma 66: 429-435, 2009.

26. Salehi M, Jalilian N, Salehi A and Ayazi M: Clinical efficacy and complications of uterine artery embolization in symptomatic uterine fibroids. Glob J Health Sci 8: 245-250, 2015.

27. Zhang E, Liu L and Owen R: Pelvic artery embolization in the management of obstetrical hemorrhage: Predictive factors for clinical outcomes. Cardiovasc Intervent Radiol 38: 1477-1486, 2015.

28. de Assis AM, Moreira AM, de Paula Rodrigues VC, Yoshinaga EM, Antunes AA, Harward SH, Srougi M and Carnevale FC: Prostatic artery embolization for treatment of benign prostatic hyperplasia in patients with prostates $>90 \mathrm{~g}$ : A prospective single-center study. J Vasc Interv Radiol 26: 87-93, 2015.

29. Al-Thunyan A, Al-Meshal O, Al-Hussainan H, Al-Qahtani MH, El-Sayed AA and Al-Qattan MM: Buttock necrosis and paraplegia after bilateral internal iliac artery embolization for postpartum hemorrhage. Obstet Gynecol 120: 468-470, 2012.

This work is licensed under a Creative Commons Attribution-NonCommercial-NoDerivatives 4.0 International (CC BY-NC-ND 4.0) License. 